\title{
Cavitation et évacuateurs de crues des grands barrages
}

\author{
par Maurice Bouvard
}

\section{I — INTRODUCTION : LES PROBLÈMES}

Une des difficultés des grands barrages correspond à l'évacuation des crues. La probabilité des débits maximum à traiter doit être à la limite nulle, car si la valeur adoptée était dépassée, la submersion de l'ouvrage qui en résulterait risquerait - surtout s'il s'agit comme c'est le cas de plus en plus fréquemment d'un massif en terre - de le détruire et de provoquer en aval une énorme onde de crue. Le débit correspondrait alors aux apports naturels en rivière augmentés de celui découlant de la vidange - accidentelle - de la retenue.

Plusieurs catégories de problèmes surgissent alors :

- Les ouvrages en cause sont essentiellement en béton. Mais ce matériau - base des ouvrages de Génie Civil présente des défauts liés aux procédés inéluctables de sa mise en œuvre. Il est alors impossible de dépasser une certaine qualité de définition des surfaces, à moins d'utiliser de très coûteux blindages pour améliorer les formes des écoulements. Ceux-ci sont alors rapidement soumis à des pressions extérieures qui exigent des ancrages coûteux et difficiles à mettre en place.

- La vitesse des écoulements est souvent très élevée : elle découle grossièrement de la charge définie par la hauteur du barrage. Mais la pression effective résulte alors d'un terme négatif très important - de l'ordre de l'énergie cinétique de 1'écoulement - comparé au terme «stabilisant » qui lui «plaque " l'eau sur le profil déversant : quelques décimètres seulement. Mais alors la moindre singularité de surface peut conduire à des pressions absolues nulles, allant jusqu'à la cavitation. L'énergie cinétique de ces types d'écoulement est par principe très élevée, alors que leur profondeur est au contraire très faible. La première risque donc très facilement de dépasser la seconde, surtout si l'on tient compte des singularités de surface dues aux imperfections du béton.

- Les formes de certains des types de vannes qui contrôlent les débits doivent évidemment tenir compte de la nécessité d'être étanches, avec une ligne de fuite de très faible longueur. Leur conception est largement définie par les conditions d'exploitation auxquelles elles sont soumises pour l'entretien, le démontage et les rechanges des systèmes d'étanchéité. Les pièces d'appui transfèrent parfois des efforts très importants, notamment sur les pièces « en coin " qui délimitent les pertuis ainsi constitués. Elles imposent plus ou moins la présence de pièces de formes peu compatibles avec le maintien d'une pression suffisante dans les écoulements (tels que les «Lignes de jet " à la partie supérieure des pertuis de fond). Des blindages de grande surface, bien définie, peuvent s'avérer indispensables pour éliminer le risque de les voir caviter, en réduisant les courbures les plus importantes des écoulements.

- Ces considérations peuvent s'avérer d'autant plus importantes que la nécessité s'impose souvent de disposer de vidanges de fond largement dimensionnées pour participer à l'évacuation des crues. Seule leur manœuvre adaptée permet de contribuer à la solution du très grave problème que constitue l'alluvionnement des retenues.

Ainsi beaucoup de détails constructifs, des problèmes aussi fondamentaux que la conception même des ouvrages pour le maintien de la capacité vive des retenues, découlent de critères fondamentaux mais souvent contradictoires.

\section{II —CROISSANCE ET DIFFICULTÉS DE L'ANALYSE DES PROBLÈMES. EXEMPLES}

Malheureusement l'analyse des problèmes ainsi soulevés s'avère souvent bien difficile. L'utilisation des modèles bute sur les difficultés liées à la multiplicité des paramètres, au rôle physique très diversifié de chacun d'eux, sur l'impossibilité d'en préciser les lois de similitude compte tenu de leur incidence très différenciée. L'influence des couches limites des écoulements sur les pressions est profonde, mais la « finesse » des considérations à la base des théories en cause n'en simplifie pas les interventions: les spécialistes du Génie Civil n'y sont guère habitués. 
Leur évolution, la turbulence des écoulements, jouent certainement un rôle fondamental dans la définition des microéléments des vitesses au voisinage immédiat des parois. On constate par exemple qu'un défaut de planéité de la surface en aval du blindage du radier d'un pertuis rectangulaire bouleverse les conditions d'érosion du métal. En ce qui concerne les surfaces bétonnées, on note deux étapes dans l'apparition des phénomènes d'érosion :

- La première, relativement lente, qui amorce le phénomène et provoque l'apparition des premières irrégularités de surface, de l'ordre de grandeur des dimensions des agrégats les plus fins du béton (le millimètre, environ).

- La seconde, où les premiers défauts ainsi provoqués se développent au contraire très vite et conduisent à un écoulement tumultueux (accompagné d'un bruit qui ne laisse aucun doute sur sa violence), impossible à définir et plus encore à contrôler. L'érosion des parois de béton croît très vite, les aciers à béton de dimension supérieure à quelque $20 \mathrm{~mm}$ sont puissamment déformés, tordus dans tous les sens. On a l'impression que la seule solution pour éviter l'apparition de phénomènes totalement incontrôlables consisterait à fermer les vannes le plus vite possible, si toutefois c'est encore possible.

Quelques exemples très particuliers permettront d'avoir une idée de la difficulté, du caractère croissant de l'importance du problème (à la mesure elle aussi croissante des débits de crues et de la hauteur des barrages).

- Le barrage de Karun, en Iran, de l'ordre de $200 \mathrm{~m}$ de hauteur [1], dans lequel l'état de surface d'un déversoir a rendu totalement inutilisable l'ouvrage, après passage d'un débit de seulement $1000 \mathrm{~m}^{3} / \mathrm{s}$ (fig. 1, 2, 3).

Les incidents survenus par cavitation dans le fonctionnement des évacuateurs de crues de l'ouvrage ont abouti à un procès intenté par le Maître d'Ouvrage (Iranien) à l'Ingénieur Conseil (Américain).

- Un des plus grands barrages du monde, Gur [2], au Vénézuéla, sur le fleuve Caroni.

On constate aussi que la « Charge critique " au-delà de laquelle la cavitation des écoulements peut apparaître est relativement faible. On l'a notée dans certains cas critiques pour des hauteurs de barrage de l'ordre de quelques dizaines de mètres (descendant même à moins de quarante mètres).

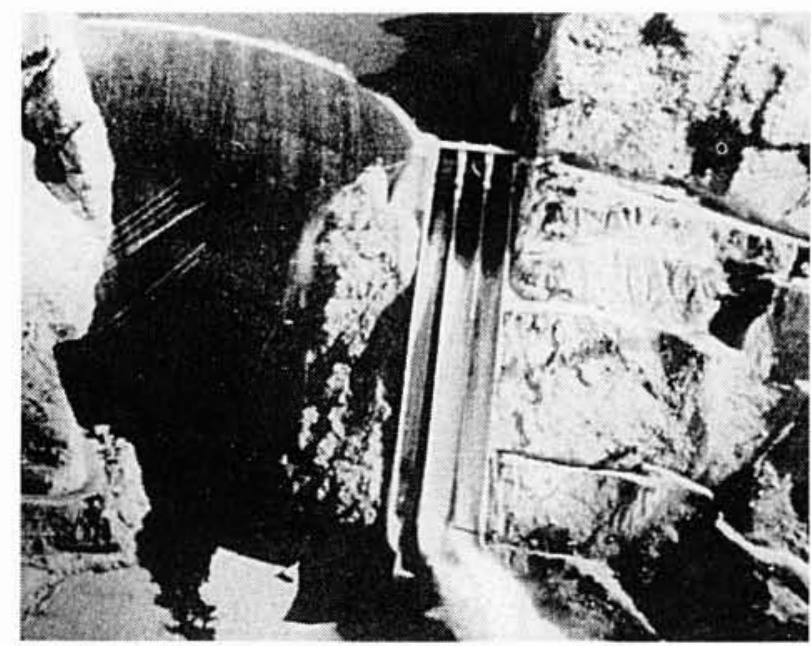

1. Barrage de Karun, vue d'ensemble, avec l'évacuateur de crues.

\section{III $\square$ SOLUTIONS}

Elles portent sur plusieurs types de phénomènes à l'origine des dégâts constatés.

- Presque dans tous les cas, l'utilisation des vannes de type wagon pour contrôler les débits des pertuis, régler les écoulements, doit absolument être prohibée. Ceux-ci sont en effet soumis à de fortes courbures, associées à de rapides changements de direction au voisinage des rainures. L'installation de vannes "Segment " présente de grands avantages, et en particulier celui de supprimer la plupart des singularités de l'écoulement (pas toutes, comme le montre l'exemple de Villerest sur la Loire), au droit et à l'aval immédiat des vannes.

- L'aération systématique des écoulements à l'aval des systèmes de contrôle des débits semble résoudre la plupart des problèmes, sous réserve de l'apport d'un débit d'air suffisant, réparti sur toute la longueur de l'enroulement, essentiellement sur le radier. Cette solution présente l'inconvénient d'en augmenter la section globale, et doit faire l'objet d'études approfondies sous les aspects théoriques et expérimentaux.
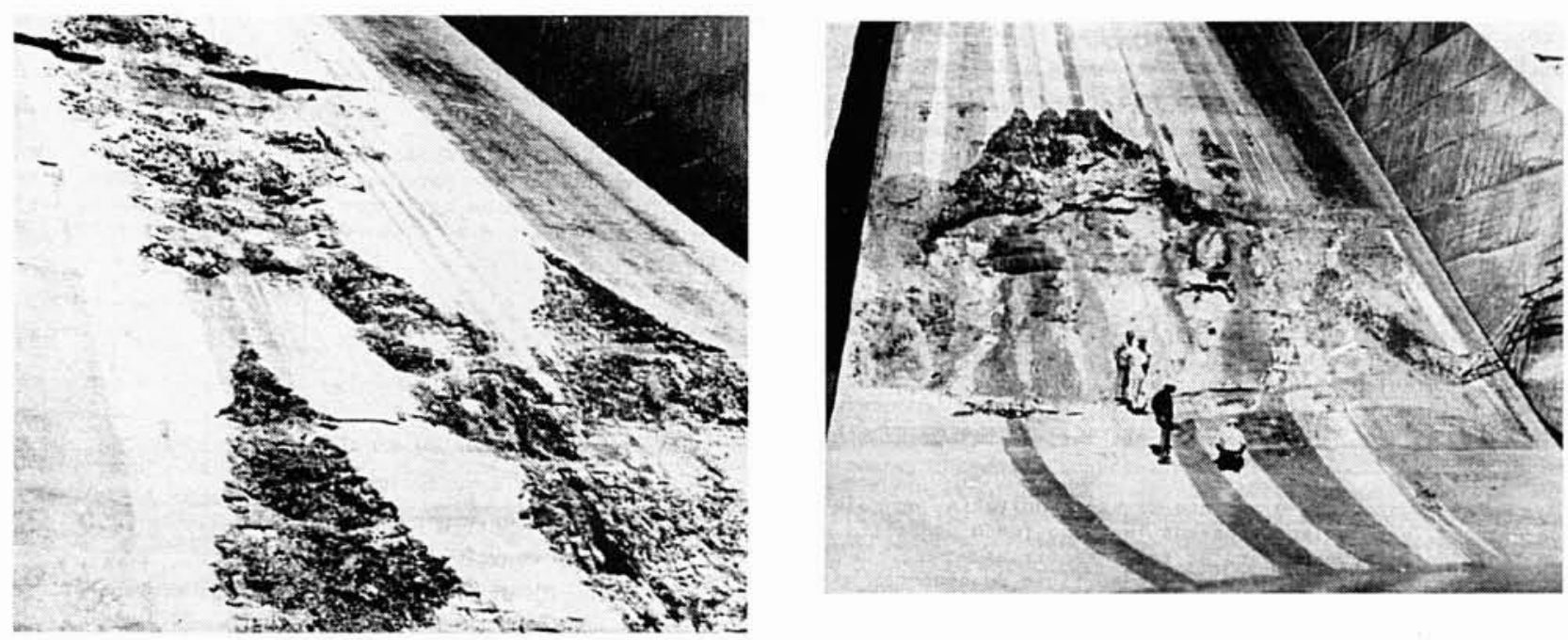

2. et 3. Barrage de Karun, érosions dues à la cavitation dans une courbe concave des pertuis du déversoir. A noter sur la figure 2 la coïncidence entre le début des usures et la présence d'un joint transversal. Remarque : l'échelle des érosions est donnée par les quatre hommes représentés sur l'une des deux photographies. 




4. Caractéristiques principales des rampes et du dispositif d'aération du système de décharge du barrage de Tarbela. (Nelson Pinto)

Ranhura do stop-log : rainure du batardeau. Blindagem: blindage. Calha de aeraçao : rainure d'aération. Parede lateral : paroi latérale.


5. Dispositif d'aération (Barrage de Foz de Areia). (Nelson Pinto)

Dispositivos de aeraçao (Barragem de Foz do Areia). Corte tipico do vertedouro: Coupe type du déversoir. Poço de Aeracao : Puits d'aération. C/L vertedouro: Axe du déversoir.

Emborcação and Foz de Areia spillways. To the Editor.

It seems worth mentioning for your records and may be of interest to your readers that both Emborcação and Foz do Areia spillways (August issue, 1983) have been subject to large floods and have performed flowlessly. Emborcação discharges have peaked a $5200 \mathrm{~m}^{3} / \mathrm{s}\left(90 \mathrm{~m}^{3} / \mathrm{s} / \mathrm{m}\right)$ after a 20-day flood averaging $2300 \mathrm{~m}^{3} / \mathrm{s}$. Foz do Areia was tested for even more severe conditions. The Iguaçu river basin was struck by an exceptionally large flood last July of recurrence estimated to be of the order of $1: 1000$ years. Foz do Areia spillway discharged more than $4000 \mathrm{~m}^{3} / \mathrm{s}$ for 28 days, the daily average flow peaking at $8240 \mathrm{~m}^{3} / \mathrm{s}\left(116.7 \mathrm{~m}^{3} / \mathrm{s} / \mathrm{m}\right)$. Both spillways were closely inspected after the floods and no damage due to cavitation was disclosed, although the maximum flow velocities had been of the order of 35 and $43 \mathrm{~m} / \mathrm{s}$ at Emborcação and Foz do Areia respectively.

Nelson Luiz de Sousa Pinto, Universidade Federal do Paraná (Extrait de "Water Power», décembre 1983).

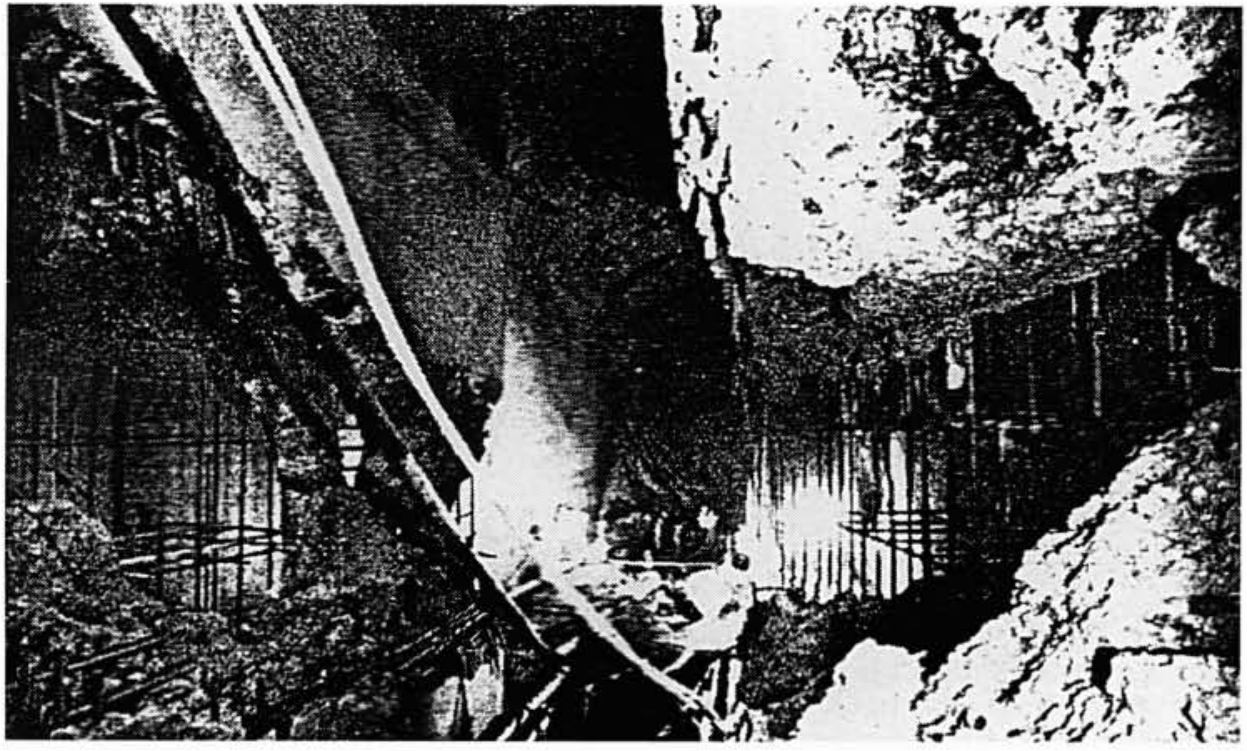

6. Tunnel 2 : dégâts sur une des piles d'entrée, vus d'amont (Tarbela). 


\section{BIBLIOGRAPHIE}

[1] Semenkov et al. - Spillway dams with aeration of the flow over spillways. Tiré à part, XI Congrès des Grands Barrages, Madrid 1973.

[2] Cavitation casts doubt on karun spillway design. World Water, juin 1979.

[3] Vidanges et évacuateurs de crues de grande capacité. $13^{\circ}$ Congrès des Grands Barrages, Question 50, New Delhi, 1979. Vidange et évacuateurs de crues de grande capacité. d) Problèmes particuliers : Evacuation des glaces, des bois, etc..., vannes à forte charge, vortex, vibrations, cavitation, etc..

[4] CHAVARri et al. - Spillway and tailrace design for raising of guri dam using large scale hydraulic model. Compte rendu Congrès CIGB, Q50 R12, New Delhi, 1979.

[5] Pinto N. - Cavitacao e aeracao em fluxo de alta velocidad. Publ. n* 35, 1979. CEHPAR, Curitiba, Brésil.
[6] Barrage de Tarbela* (fig. 4 et 6).

[7] NeIDERT H. - Desempenho de vertedores. Dissipacao de energia. Cavitacao e aeracao. Publ. n*37, 1980. CEHPAR, Brésil.

[8] Chanson H. - Aération d'un jet libre sur un évacuateur de crues. Journal of Hydraulic Research, n 5, 1991.

[8 bis] Chanson H. - Prévision du remplissage des cavités ventilées derrière des aérateurs d'évacuateurs de crues. Journal of Hydraulic Research, n 3, 1995. M. Chanson, Maître de Conférences à l'Université de Queensland, est ancien élève de l'Ecole (Pr 1983).

(*) Le plus grand barrage du monde. Le volume de l'ouvrage (En terre) atteint 130 millions de $\mathrm{m}^{3}$, pour une retenue de 1,36 milliards de $\mathrm{m}^{3}$. Sa construction, sa mise en service et son exploitation depuis 20-30 ans se sont traduits par une séquence presque invraisemblable d'incidents dus essentiellement à des phénomènes hydrauliques (associés à la mauvaise qualité des fondations), notamment de cavitation dans les ouvrages annexes du barrage, dations), notamment de cavitation dans les ouvrages annexes du barrage,
particulièrement importants (capacité des évacuateurs de crues très voisine de $40000 \mathrm{~m}^{3} / \mathrm{s}$ ) 